\title{
Existence of Compactly Supported Global Minimisers for the Interaction Energy
}

\author{
José A. Cañizo, José A. Carrillo \& Francesco S. Patacchini
}

\author{
Communicated by I. FonSECA
}

\begin{abstract}
The existence of compactly supported global minimisers for continuum models of particles interacting through a potential is shown under almost optimal hypotheses. The main assumption on the potential is that it is catastrophic, or not H-stable, which is the complementary assumption to that in classical results on thermodynamic limits in statistical mechanics. The proof is based on a uniform control on the local mass around each point of the support of a global minimiser, together with an estimate on the size of the "gaps" it may have. The class of potentials for which we prove the existence of global minimisers includes power-law potentials and, for some range of parameters, Morse potentials, widely used in applications. We also show that the support of local minimisers is compact under suitable assumptions.
\end{abstract}

\section{Introduction and Main Results}

The analysis of configurations minimising nonlocal interaction energies is an ubiquitous question in mathematics with applications ranging from physics and engineering problems to mathematical biology and game theory in economic and social sciences. Understanding the balance of the effects of interactions between the "particles" in applications such as inelastic particles in granular flows [8, 22, 23, 39], molecules in self-assembly materials and virus structures [28,37,42,49], animals in flock patterns in biological swarms $[19,20,47,48]$, and individuals' strategies in pedestrian dynamics or strategic preferences $[12,26]$, is of paramount importance.

We deal with interaction potentials: $W: \mathbb{R}^{d} \rightarrow \mathbb{R} \cup\{+\infty\}$ is a pointwise defined and measurable function which is allowed to take the value $+\infty$, and whose gradient models the interaction force between two particles located at a distance $x \in \mathbb{R}^{d}$. More precisely, we regard $-\nabla W(y-x)$ as the force that a particle at $x$ exerts on a particle at $y$, and accordingly we say that $W$ is attractive at $x \in \mathbb{R}^{d}$ when $-\nabla W(x) \cdot x \leqq 0$, and repulsive when $-\nabla W(x) \cdot x \geqq 0$. Given $N$ particles with 
positions $x_{i} \in \mathbb{R}^{d}$, for any $i \in\{1, \ldots, N\}$, we can define the energy associated with them as

$$
E_{N}\left(x_{1}, \ldots, x_{N}\right)=\frac{1}{2 N^{2}} \sum_{i, j=1}^{N} W\left(x_{i}-x_{j}\right) .
$$

The typical potentials that we have in mind are repulsive at short distances (that is for $|x|<r$ for some $r>0$ ) and attractive at large ones, and it is the interplay of these two effects that allows for the existence of minimisers with interesting properties. The minimisers of the discrete energy should realise the most stable balance between the possible attractive and repulsive effects encoded in the interaction potential $W$. The normalisation of the discrete energy is done in such a way that it kept of order one as $N \rightarrow \infty$. Finding global (and local) minimisers of this discrete energy $E_{N}$ is a question of major interest in crystallisation, where self-interaction is avoided, that is $W(0)=0$ (see [46] and the references therein), but also for less singular potentials where the normalised discrete minima may converge towards some integrable density or non-atomic probability measure when $N \rightarrow \infty$.

It is therefore more suitable to relax the variational problem and look for global minimisers of the interaction energy functional $E: \mathcal{P}\left(\mathbb{R}^{d}\right) \rightarrow \mathbb{R} \cup\{+\infty\}, d \geqq 1$, defined on the set $\mathcal{P}\left(\mathbb{R}^{d}\right)$ of probability measures on $\mathbb{R}^{d}$ by

$$
E(\rho)=\frac{1}{2} \int_{\mathbb{R}^{d} \times \mathbb{R}^{d}} W(x-y) \mathrm{d} \rho(x) \mathrm{d} \rho(y), \quad \rho \in \mathcal{P}\left(\mathbb{R}^{d}\right) .
$$

If one considers $\rho$ as a given mass distribution, then (1.1) is its total potential energy when individual particles interact through a pair potential $W$ (that is the potential energy of two particles with unit mass, one at $x \in \mathbb{R}^{d}$ and one at $y \in \mathbb{R}^{d}$, is $W(x-y))$. More precisely, in the following, if $\rho \in \mathcal{P}\left(\mathbb{R}^{d}\right)$ satisfies $E(\rho) \leqq E(\mu)$ for all $\mu \in \mathcal{P}\left(\mathbb{R}^{d}\right)$ we say that $\rho$ is a ground state or global minimiser of $E$ (and sometimes simply minimiser). Analogously, we talk about minimisers on a subset $\mathcal{A} \subseteq \mathcal{P}\left(\mathbb{R}^{d}\right)$ when the inequality holds in $\mathcal{A}$.

Let us mention that the set of global (and local) minimisers of the total potential energy for not too singular potentials can be very rich in terms of their qualitative properties depending on the behaviour of the potential at the origin. Actually, there are plenty of works reporting on the qualitative properties of critical points of the discrete energy in the context of collective behaviour (see [27,47,48] and the references therein). For instance, Morse potentials were considered in [27], that is, potentials of the form

$$
W(x)=C_{R} e^{-\frac{|x|}{\ell_{R}}}-C_{A} e^{-\frac{|x|}{\ell_{A}}}, \quad x \in \mathbb{R}^{d},
$$

with $C_{R}, C_{A}$ measuring the strengths of the repulsive and the attractive part, respectively, and $\ell_{R}, \ell_{A}$ being the typical lengths scales for repulsion and attraction, respectively. The authors' detailed numerical study indicates that these potentials lead to patterns corresponding to minimisers of the energy as $N \rightarrow \infty$ only in the range of parameters corresponding to $\ell_{R}<\ell_{A}$ and $C_{R} / C_{A}<\left(\ell_{A} / \ell_{R}\right)^{d}$. Furthermore, they noted that these conditions are intimately related to the classical notion of $H$-stability in statistical mechanics. 
Before discussing this connection further, we now introduce the set of hypotheses on the interaction potential needed to state our main results in full rigour. We always assume, without loss of generality, that

Hypothesis 1. $W$ is bounded from below by a finite constant $W_{\min }<0$.

This ensures that (1.1) is well defined (possibly equal to $+\infty$ ) for all $\rho \in \mathcal{P}\left(\mathbb{R}^{d}\right)$. Actually, in order for $E$ to be finite on "nice" probability measures $\rho$ (say, uniformly distributed on a ball), we also assume

Hypothesis 2. $W$ is locally integrable (that is, $\int_{B}|W|<+\infty$ for any open ball $B \subset \mathbb{R}^{d}$ ).

In particular, Hypothesis 2 implies that the potential $W$ cannot be equal to $+\infty$ on sets with positive Lebesgue measure. This assumption rules out interaction potentials which are too singular at the origin as those used in the analysis of the crystallisation phenomena $[29,46]$ (see further comments before Hypothesis 4 below).

Since we think of $W$ as a potential as explained above, it is natural to assume additionally

Hypothesis 3. $W$ is symmetric (that is, $W(x)=W(-x)$ for all $x \in \mathbb{R}^{d}$ ).

It is also quite natural to assume that the potential $W$ is radial, but since we do not need that in the following we avoid making the assumption.

The problem of finding global minimisers of (1.1) has two fundamental invariances. First, $E$ is invariant under translations: for all $\rho \in \mathcal{P}\left(\mathbb{R}^{d}\right)$ and $z \in \mathbb{R}^{d}$ we have $E(\rho)=E\left(T_{z}(\rho)\right)$, where $T_{z}(\rho)$ is the push-forward of $\rho$ by the $z$-translation $T_{z}: \mathbb{R}^{d} \rightarrow \mathbb{R}^{d}, x \mapsto x-z$, that is $T_{z}(\rho)(x)=\rho(x+z)$ for integrable densities. In particular, any translation of a minimiser is also a minimiser, and uniqueness (if it holds) can only be expected up to translations. Second, if we add an arbitrary constant $D \in \mathbb{R}$ to $W$, then the energy $E$ is shifted by $\frac{1}{2} D$, and hence the minimisation problem does not change (note that this is consistent with the interpretation of $W$ as a potential, which is arbitrary up to a constant).

Our main motivation for studying these minimisers has mainly come from the recent interest in the field of collective behaviour regarding the steady states of the aggregation equation

$$
\partial_{t} \rho=\nabla \cdot(\rho(\nabla W * \rho)),
$$

where $\rho=\rho(t, x)$ is a function (possibly a measure) defined for $t \geqq 0$ and $x \in \mathbb{R}^{d}$. Since $E$ is a Lyapunov functional for Equation (1.3) (in fact, (1.3) is the gradient flow of $E$ with respect to the Wasserstein transport distance [17,22,23]) its minimisers (if they exist) are natural candidates for a steady state of (1.3), and they are also natural candidates to represent the typical asymptotic behaviour of (1.3) as $t \rightarrow+\infty$. Equations of the form (1.3) appear in granular flow [22,23,39] and in swarming models (see $[9,10,38]$ and the references therein) as mentioned before.

It is easy to see that if a minimiser $\rho$ happens to be regular enough then it must satisfy the corresponding Euler-Lagrange equation, namely

$$
W * \rho=C \text { on } \operatorname{supp} \rho,
$$


for some $C \in \mathbb{R}$ (see Lemma 2.3 and Remark 2.4 for a more precise statement). Consequently, it must be a stationary state of (1.3), again assuming that $\rho$ is regular enough for the right-hand side of (1.3) to be meaningful. Equation (1.4) also appears as a condition satisfied by special solutions in a variety of models (for example, flock solutions in $[1,19,20,27])$, and it is interesting in itself.

As we already mentioned, the authors in [27] analysed numerically a discrete collective behaviour model based on the Morse potentials of the form (1.2), and it was noticed that its large-time asymptotics seemed to depend on whether the potential satisfied a classical condition known as stability or H-stability in classical statistical mechanics $[34,43]$ :

Definition 1.1. (Stability) Take a potential $W: \mathbb{R}^{d} \rightarrow \mathbb{R} \cup\{+\infty\}$ satisfying Hypotheses 1 and 2 , and assume that its limit at $\infty$ exists (being possibly equal to $+\infty)$. Let us define

$$
W_{\infty}:=\lim _{|x| \rightarrow \infty} W(x) .
$$

We say that $W$ is stable if

$$
E(\rho) \geqq \frac{1}{2} W_{\infty} \text { for all } \rho \in \mathcal{P}\left(\mathbb{R}^{d}\right) .
$$

Otherwise we say that $W$ is unstable. In a similar way, we define the concept of stability/instability on a subset $\mathcal{A} \subseteq \mathcal{P}\left(\mathbb{R}^{d}\right)$ by restricting (1.6) to all $\rho \in \mathcal{A}$.

The term catastrophic instead of unstable is also used in part of the statistical mechanics literature, where a common assumption is that $W(x) \rightarrow 0$ as $|x| \rightarrow \infty$; in that case Equation (1.6) translates to $E(\rho) \geqq 0$ for all $\rho \in \mathcal{P}\left(\mathbb{R}^{d}\right)$, which is the definition often given classically (see for example [7, eq. (23)]). The notion of stability given in Definition 1.1 implies the classical notion of stability if the potential is such that $W(0)$ is finite (see for example [43, Sections 3.1 and 3.2]). In statistical mechanics the classical condition of stability is motivated by the fact that, when combined with that of temperedness, it is sufficient to show the thermodynamic behaviour of a system of particles interacting via a pairwise potential (see [43, Theorem 3.3.12]). Indeed, the former condition avoids that infinitely many particles collapse in a bounded region and the latter avoids significant interaction between distant particles.

Last but not least, the crystallisation phenomena discussed in $[29,46]$ (see [2,35] for other related work) are closely linked to the problem we consider, though the energy there is minimised among configurations of a finite number of particles, and does not include their self-interaction. The results in these references give more detailed information about the behaviour of the minimisers for large number of particles in particular stable cases. Essentially, the minimisers form regular lattices which spread and fill the whole space as the number of particles tends to infinity. In other words, if we insist in normalising the minimisers as probability measures, these Dirac delta minimisers tend to zero weakly as measures as the number of particles tends to infinity. In fact, the infimum of the interaction energy (modified as mentioned above to exclude self-interactions) over linear combinations of Dirac deltas is zero but is not attained. 
By contrast, in our setting we need to assume that $W$ is unstable in order to show the existence of a minimiser:

Hypothesis 4. The limit (1.5) exists and $W$ is unstable.

We also assume the following more technical hypotheses, though some of our results do not require Hypothesis 6:

Hypothesis 5. $W$ is lower semi-continuous.

Hypothesis 6. There is $R_{6}>0$ such that $W$ is strictly increasing on $\mathbb{R}^{k-1} \times$ $\left[R_{6}, \infty\right) \times \mathbb{R}^{d-k}$ as a function of its $k$-th variable, for all $k \in\{1, \ldots, d\}$.

Remark 1.2. Notice that any potential which is radially strictly increasing outside some ball (that is for some $R_{6}>0$ we have $W(x)>W(y)$ whenever $|x|>|y| \geqq$ $R_{6}$ ) satisfies Hypothesis 6.

Remark 1.3. Hypothesis 6 is unnecessary in order to show the existence of global minimisers (see the related results in [45]). However, some growth condition such as Hypothesis 6 seems to be necessary to show the uniform compactness part of Theorem 1.4 below (see Remark 1.6). An example of a growth condition which we could use here that is less restrictive (but also less intuitive) than Hypothesis 6 is given in Remark 2.8.

Our main result is the following:

Theorem 1.4. Assume that $W: \mathbb{R}^{d} \rightarrow \mathbb{R} \cup\{+\infty\}$ satisfies Hypotheses $1-6$. Then there exists a global minimiser $\rho \in \mathcal{P}\left(\mathbb{R}^{d}\right)$ of the energy $E$. In addition, there exists $K>0$ (depending only on $W$ and the dimension $d$ ) such that every minimiser of $E$ has compact support with diameter at most $K$.

We point out that an explicit estimate on the size of the support can be recovered from the proof of the above theorem in Section 2, though we do not expect it to be sharp.

Our proof of Theorem 1.4 rests on two key apriori estimates on minimisers. First, in Lemma 2.6 we show that any point in the support of a minimiser needs to have at least a fixed amount $m>0$ of mass which is not further away than a fixed distance $r>0$. The intuitive reason for this is that the potential energy $W * \rho$ has to be constant at $\rho$-almost every point of the support of a minimiser $\rho$ (cf. Lemma 2.3 and [3, Theorem 4(i)]), and that potential energy has to be strictly less than the "potential at infinity" $W_{\infty}$ due to the instability assumption in Hypothesis 4 . Hence some mass has to be close to any given point $x$ in the support, since mass being too far away would mean that $W * \rho(x)$ is too close to $W_{\infty}$. Secondly, Lemma 2.7 uses Hypothesis 6 to show that the support of a minimiser cannot have arbitrarily large "gaps". Otherwise one could bring closer the two parts of the minimiser separated by the gap and obtain a mass distribution with a smaller energy. This, together with the first estimate, shows that a minimiser has to consist of at most $\lceil 1 / \mathrm{m}\rceil$ pieces, each with mass at least $m$, not too far apart from each other, where $\lceil\cdot\rceil$ is the ceiling function. An apriori estimate on the size of the support is then easily obtained. 
The proof of Theorem 1.4 is then completed by a usual approximation argument: we consider minimisers $\rho_{R}$ among the set of measures supported in $\bar{B}(0, R)$, the closed ball of centre 0 and radius some $R$, and show that these estimates hold uniformly for them, which allows us to pass to the limit as $R \rightarrow+\infty$ to obtain a global minimiser.

We have recently learnt that Simione et al. [45] independently proved a similar result by a different method based on Lions' concentration compactness principle while this paper was being prepared. Their method does not give any estimate on the support or properties of the minimisers; on the other hand, their conditions for existence of minimisers are slightly sharper than the ones in Theorem 1.4. To complement their result we give a corollary which derives directly from the structure of our proof of Theorem 1.4. It is proved in Section 2.4 below.

Corollary 1.5. Assume that $W: \mathbb{R}^{d} \rightarrow \mathbb{R} \cup\{+\infty\}$ satisfies Hypotheses $1-5$ (satisfied by any potential considered in [45] as long as it is unstable in our sense). Suppose moreover that there exists a global minimiser $\rho \in \mathcal{P}\left(\mathbb{R}^{d}\right)$ of the energy (1.1). Then $\rho$ is compactly supported.

Remark 1.6. We emphasise that Corollary 1.5 does not require Hypothesis 6 on the growth at $\infty$ of the potential $W$. Hence global minimisers for potentials which, for instance, decrease to 0 at $\infty$ and satisfy Hypotheses $1-5$, have to be compactly supported. Note that our statement in this case does not show the existence of any uniform bound $K$ on the size of the support of a global minimiser (unlike Theorem 1.4).

Local minimisers of the energy (1.1) in several transport distances (that is minimisers in a small ball around them) were studied in [3], where bounds on the dimension of their support were given under some assumptions controlling the strength of the repulsion at the origin. Moreover, Euler-Lagrange conditions for local minimisers in several transport distances were also obtained. These results (see [3, Theorem 4]) apply to our case since a global minimiser is of course a local one in particular. Note that, in Section 2.5 below, we derive a generalisation of Corollary 1.5 to $d_{2}$-local minimisers, where $d_{2}$ is the quadratic Wasserstein distance. Let us mention that the rich structure of global and local minimisers of the interaction energy was shown for several potentials and by different numerical methods in [1,3-5, 15,20,32,33,47].

Let us review some of the known rigorous results on the existence, uniqueness, and other properties of these minimisers in some remarkable cases. An often studied case is that in which the potential $W$ is a sum of powers:

$$
W(x)=\frac{|x|^{a}}{a}-\frac{|x|^{b}}{b}, \quad x \in \mathbb{R}^{d},
$$

for some $a, b \in \mathbb{R}$ with $-d<b<a$, and the understanding that $|x|^{0} / 0 \equiv \log |x|$. Here the term $|x|^{a} / a$ is the attractive one (being an increasing function of $|x|$, regardless of the sign of $a$ ), and $|x|^{b} / b$ is the repulsive one (since it is a decreasing function of $|x|$ ). For $b=2-d$ the repulsive term is called the Newtonian potential. It is not difficult to check that this class of potentials satisfies our hypotheses (see Section 3). 
The case $a=2$ simplifies the problem a lot since the attractive part of the interaction can be reduced to an external quadratic confinement by expanding the square. The case $b=2-d, a=2$ is actually relatively well-known among probabilists: up to translations, the unique global minimiser is the characteristic of a ball with an appropriate radius. A closely related result with a compact confinement is proved in [36], and the 2-dimensional case can be found for example in [44, Theorem 6.1, p. 245]. The extension to higher dimensions can be found in [40, Proposition 2.13]. The interest in this problem on these references comes from its links to the capacity of sets and applications to random matrix theory (see [24] and the references therein).

A modified minimisation problem for power-law potentials with $a>-d$ was recently studied in [25], where the authors showed that there exists a minimiser $\rho_{M}$ in the class of radially symmetric functions with a fixed $L^{\infty}$-bound $M$. They also show that for $-d<a<0$ the condition of radial symmetry is not needed, so that in that case a minimiser exists in the class of functions with a given $L^{\infty}$-bound.

The case $a=2, b=2 s-d$ for $0<s<1$ was studied in [13] in relation to the asymptotic behaviour of Equation (1.3), referred to as the fractional porous medium equation. The authors there showed that there is a unique steady state (up to translations) to (1.3), which they called a modified Barenblatt profile. Since the uniqueness was shown via the associated obstacle problem and the Euler-Lagrange conditions in [3, Theorem 4] show that global minimisers are regular solutions of the obstacle problem (see also [16]), then their uniqueness result implies the uniqueness of global minimisers. Finally, all cases with $-d<b \leqq 2-d$ and $a>0$ were recently treated in [16] showing the regularity of local minimisers using the connection to classical obstacle problems, by methods that can treat more general potentials than power laws: results in [16] apply to potentials behaving like $-|x|^{b} / b$ at zero in the range $-d<b \leqq 2-d$ with a smooth enough attractive part of the potential.

On the other hand, there have been many works devoted to the study of the steady states and long-time behaviour of Equation (1.3) (see [3-5, 17, 18,30-33,41] and the references therein). Steady states for the case $b=2-d, a>0$ were studied in $[32,33]$, where it was proved that there exists a unique radial compactly supported steady state (up to translations). The asymptotic behaviour of (1.3) in the case $b=2-d, a=2$ was studied in [11,32], and the case $-d<b<2-d, a=2$, as already remarked, was considered in $[13,14]$.

Finally, other particularly interesting potentials are Morse-like potentials [5, $9,19,21,27]$ (treated in Section 3), for which there is a huge amount of numerical evidence regarding the existence of compactly supported global minimisers. To our knowledge, a proof of this existence was not previously available.

The paper is organised as follows: in Section 2 we prove Theorem 1.4. We split the proof of the existence part into three main steps: first we show the existence of global minimisers on each set of probability measures supported on a given ball, second we prove that the support diameters of such minimisers are uniformly bounded, and third we show existence on the whole space $\mathcal{P}\left(\mathbb{R}^{d}\right)$. At the end of this section we give the generalisation of Corollary 1.5 to local minimisers with 
respect to the quadratic Wasserstein topology. In Section 3 we give examples of potentials satisfying the hypotheses of Theorem 1.4, as well as conditions for the non-existence of global minimisers which show that Hypothesis 4 in Theorem 1.4 is almost sharp.

\section{Existence of Minimisers}

\subsection{Minimisers on a Given Ball}

Let us define for all $R \geqq 0$ the $\operatorname{set} \mathcal{P}_{R}\left(\mathbb{R}^{d}\right)=\left\{\rho \in \mathcal{P}\left(\mathbb{R}^{d}\right) \mid \operatorname{supp} \rho \subset \bar{B}(0, R)\right\}$. This is the set of all probability measures with support included in the closed ball $\bar{B}(0, R)$. For every $R \geqq 0$, we want to show the existence of global minimisers on $\mathcal{P}_{R}\left(\mathbb{R}^{d}\right)$.

Let us first show the following lemma:

Lemma 2.1. Let $W$ be a potential satisfying Hypotheses 1 and 5. Then the energy (1.1) is weakly- $\star$ lower semi-continuous, that is for any sequence $\left(\rho_{n}\right)_{n \in \mathbb{N}}$ converging weakly $\star$ to $\rho$ in $\mathcal{P}\left(\mathbb{R}^{d}\right)$ we have $E(\rho) \leqq \lim _{n \rightarrow \infty} E\left(\rho_{n}\right)$.

Proof. By Hypotheses 1 and 5 we know that there exists a non-decreasing sequence $\left(\varphi_{m}\right)_{m \in \mathbb{N}}$ of continuous and bounded functions such that $\varphi_{m} \rightarrow W$ pointwise as $m \rightarrow \infty$ (see [6, Lemma A.1.3]). Let us consider a bound from below for $\varphi_{1}$, and denote it by $c$. Then $\left(\varphi_{m}-c\right)_{m \in \mathbb{N}}$ is non-negative, non-decreasing and with pointwise limit $W-c$. Suppose that $\left(\rho_{n}\right)_{n \in \mathbb{N}}$ is a sequence weakly- $\star$ converging to $\rho$ in $\mathcal{P}\left(\mathbb{R}^{d}\right)$. Then, applying the Lebesgue monotone convergence theorem, we obtain

$\int_{\mathbb{R}^{d} \times \mathbb{R}^{d}}\left(\varphi_{m}(x-y)-c\right) \mathrm{d} \rho(x) \mathrm{d} \rho(y) \underset{m \rightarrow \infty}{\longrightarrow} \int_{\mathbb{R}^{d} \times \mathbb{R}^{d}}(W(x-y)-c) \mathrm{d} \rho(x) \mathrm{d} \rho(y)$.

Therefore, we infer

$$
\int_{\mathbb{R}^{d} \times \mathbb{R}^{d}} \varphi_{m}(x-y) \mathrm{d} \rho(x) \mathrm{d} \rho(y) \underset{m \rightarrow \infty}{\longrightarrow} \int_{\mathbb{R}^{d} \times \mathbb{R}^{d}} W(x-y) \mathrm{d} \rho(x) \mathrm{d} \rho(y) .
$$

Furthermore, since $\left(\varphi_{m}\right)_{m \in \mathbb{N}}$ is non-decreasing, we have for all $n, m \in \mathbb{N}$,

$$
\int_{\mathbb{R}^{d} \times \mathbb{R}^{d}} \varphi_{m}(x-y) \mathrm{d} \rho_{n}(x) \mathrm{d} \rho_{n}(y) \leqq \int_{\mathbb{R}^{d} \times \mathbb{R}^{d}} W(x-y) \mathrm{d} \rho_{n}(x) \mathrm{d} \rho_{n}(y) .
$$

Hence, by definition of weak- $\star$ convergence and passing to the limits $n, m \rightarrow \infty$, we get

$$
\int_{\mathbb{R}^{d} \times \mathbb{R}^{d}} W(x-y) \mathrm{d} \rho(x) \mathrm{d} \rho(y) \leqq \liminf _{n \rightarrow \infty} \int_{\mathbb{R}^{d} \times \mathbb{R}^{d}} W(x-y) \mathrm{d} \rho_{n}(x) \mathrm{d} \rho_{n}(y),
$$

proving the desired result.

We can now state an existence result for global minimisers of the energy (1.1) on $\mathcal{P}_{R}\left(\mathbb{R}^{d}\right)$ for any $R \geqq 0$. 
Lemma 2.2. Suppose that the potential $W$ satisfies Hypotheses 1 and 5. Then for every $R \geqq 0$ there exists a global minimiser $\rho_{R}$ on $\mathcal{P}_{R}\left(\mathbb{R}^{d}\right)$ of the energy (1.1).

Proof. Let us fix $R \geqq 0$ and let $\left(\rho_{n}\right)_{n \in \mathbb{N}}$ be a minimising sequence for the restriction of the energy (1.1) to $\mathcal{P}_{R}\left(\mathbb{R}^{d}\right)$. Note that $\mathcal{P}_{R}\left(\mathbb{R}^{d}\right)$ is a tight subset of $\mathcal{P}\left(\mathbb{R}^{d}\right)$, and therefore by Prohorov's theorem we know there exists a subsequence $\left(\rho_{n_{k}}\right)_{k \in \mathbb{N}}$ and $\rho_{R}$ such that $\rho_{n_{k}} \rightarrow \rho_{R}$ weakly- $\star$ as $k \rightarrow \infty$. Moreover, since $\mathcal{P}_{R}\left(\mathbb{R}^{d}\right)$ is weakly- $\star$ closed, we have $\rho_{R} \in \mathcal{P}_{R}\left(\mathbb{R}^{d}\right)$. By Lemma 2.1 we have $\lim \inf _{k \rightarrow \infty} E\left(\rho_{n_{k}}\right) \geqq$ $E\left(\rho_{R}\right)$. Hence, $\inf \left\{E(\rho) \mid \rho \in \mathcal{P}_{R}\left(\mathbb{R}^{d}\right)\right\}=\lim _{n \rightarrow \infty} E\left(\rho_{n}\right)=\liminf _{k \rightarrow \infty} E\left(\rho_{n_{k}}\right)$ $\geqq E\left(\rho_{R}\right) \geqq \inf \left\{E(\rho) \mid \rho \in \mathcal{P}_{R}\left(\mathbb{R}^{d}\right)\right\}$. Therefore $\rho_{R}$ is a global minimiser of the energy (1.1) on $\mathcal{P}_{R}\left(\mathbb{R}^{d}\right)$.

\subsection{Uniform Bound on the Support of Minimisers}

We now show the existence of a bound for the diameter of the support of global minimisers in $\mathcal{P}_{R}\left(\mathbb{R}^{d}\right)$ for any $R \geqq 0$, which is uniform in $R$.

In [3, Theorem 4(i)] it was proved that a global minimiser $\rho$ satisfies $W * \rho=$ $2 E(\rho) \rho$-almost everywhere. We adapt it here for minimisers on $\mathcal{P}_{R}\left(\mathbb{R}^{d}\right)$ for any $R \geqq 0$ :

Lemma 2.3. Assume that the potential $W$ satisfies Hypotheses 1-3 and 5. Take $R \geqq 0$ and let $\rho_{R}$ be a global minimiser on $\mathcal{P}_{R}\left(\mathbb{R}^{d}\right)$. Then $W * \rho_{R}=2 E\left(\rho_{R}\right)$ $\rho_{R}$-almost everywhere.

Proof. Let $\varphi \in C_{0}^{\infty}\left(\mathbb{R}^{d}\right)$ and define

$$
\mathrm{d} \nu(x)=\left(\varphi(x)-\int_{\mathbb{R}^{d}} \varphi(y) \mathrm{d} \rho_{R}(y)\right) \mathrm{d} \rho_{R}(x)
$$

for all $x \in \mathbb{R}^{d}$. Also consider $\rho_{\varepsilon}=\rho_{R}+\varepsilon v$ for $\varepsilon>0$. Then we have

$$
\int_{\mathbb{R}^{d}} \mathrm{~d} \rho_{\varepsilon}(x)=\int_{\mathbb{R}^{d}} \mathrm{~d} \rho_{R}(x)+\varepsilon \int_{\mathbb{R}^{d}} \mathrm{~d} \nu(x)=1 .
$$

Moreover, one can check that

$$
\varphi(x)-\int_{\mathbb{R}^{d}} \varphi(y) \mathrm{d} \rho_{R}(y) \geqq-2\|\varphi\|_{L^{\infty}\left(\mathbb{R}^{d}\right)} .
$$

Thus, for any Borel set $A$ of $\mathbb{R}^{d}$ we have

$$
\begin{aligned}
\rho_{\varepsilon}(A) & =\int_{A} \mathrm{~d} \rho_{\varepsilon}(x) \geqq \int_{A} \mathrm{~d} \rho_{R}(x)-2 \varepsilon\|\varphi\|_{L^{\infty}\left(\mathbb{R}^{d}\right)} \rho_{R}(A) \\
& =\left(1-2 \varepsilon\|\varphi\|_{L^{\infty}\left(\mathbb{R}^{d}\right)}\right) \rho_{R}(A) .
\end{aligned}
$$

Therefore, since $\rho_{R}$ is a probability measure,

$$
\rho_{\varepsilon}(A) \geqq 0 \text { if } \varepsilon \leqq \frac{1}{2\|\varphi\|_{L^{\infty}\left(\mathbb{R}^{d}\right)}} .
$$


Let us take such an $\varepsilon$, which ensures that $\rho_{\varepsilon}$ is a probability measure. Furthermore, we have $\operatorname{supp} \rho_{\varepsilon} \subset \bar{B}(0, R)$. Hence $\rho_{\varepsilon} \in \mathcal{P}_{R}\left(\mathbb{R}^{d}\right)$. We know that $\rho_{R}$ is a global minimiser on $\mathcal{P}_{R}\left(\mathbb{R}^{d}\right)$, and thus $E\left(\rho_{\varepsilon}\right) \geqq E\left(\rho_{R}\right)$. In addition, by Hypotheses 1 and 2 the energy generated by $\rho_{R}$ is bounded. Then

$$
\begin{aligned}
\frac{E\left(\rho_{\varepsilon}\right)-E\left(\rho_{R}\right)}{\varepsilon}= & \int_{\mathbb{R}^{d} \times \mathbb{R}^{d}} W(x-y) \mathrm{d} \nu(x) \mathrm{d} \rho_{R}(y) \\
& +\frac{\varepsilon}{2} \int_{\mathbb{R}^{d} \times \mathbb{R}^{d}} W(x-y) \mathrm{d} \nu(x) \mathrm{d} \nu(y) \geqq 0 .
\end{aligned}
$$

Hence, letting $\varepsilon \rightarrow 0$ and since the last integral is finite, we get

$$
\int_{\mathbb{R}^{d} \times \mathbb{R}^{d}} W(x-y) \mathrm{d} \nu(x) \mathrm{d} \rho_{R}(y) \geqq 0,
$$

or equivalently, by plugging the definition of $v$ inside the integral,

$$
\int_{\mathbb{R}^{d}}\left(W * \rho_{R}(x)-2 E\left(\rho_{R}\right)\right) \varphi(x) \mathrm{d} \rho_{R}(x) \geqq 0 .
$$

This result being true for all $\varphi \in C_{0}^{\infty}\left(\mathbb{R}^{d}\right)$, we have that $W * \rho_{R}(x)-2 E\left(\rho_{R}\right)=0$ $\rho_{R}$-almost everywhere.

Remark 2.4. By following the same argument one can see that Lemma 2.3 is also true for a global minimiser $\rho$ on the whole space $\mathcal{P}\left(\mathbb{R}^{d}\right)$ (this is the content of $[3$, Theorem 4(i)]).

To show that, under the instability condition in Hypothesis 4 , the diameter of the support of a global minimiser $\rho_{R}$ on $\mathcal{P}_{R}\left(\mathbb{R}^{d}\right)$ is independent of $R$, we first notice that an unstable potential is also unstable on $\mathcal{P}_{S}\left(\mathbb{R}^{d}\right)$ for some finite radius $S$ :

Lemma 2.5. Assume Hypotheses 1 and 4 for the potential $W$. Then there exists $S>0$ such that $W$ is unstable on $\mathcal{P}_{S}\left(\mathbb{R}^{d}\right)$ (and hence on $\mathcal{P}_{R}\left(\mathbb{R}^{d}\right)$ for all $R \geqq S$ ).

Proof. Let $\rho \in \mathcal{P}\left(\mathbb{R}^{d}\right)$ be such that $E(\rho)<\frac{1}{2} W_{\infty}$. Define the sequence of truncated probabilities $\left(\rho_{n}\right)_{n} \subset \mathcal{P}\left(\mathbb{R}^{d}\right)$, for every $n \in \mathbb{N}$ large enough so that $\rho(B(0, n))>0$, by

$$
\rho_{n}=\frac{1}{\rho(B(0, n))} \chi_{B(0, n)} \rho .
$$

(Where $\chi_{A}$ denotes the characteristic function of a set $A$.) Clearly, for every such $n$, we have $\rho_{n} \in \mathcal{P}_{n}\left(\mathbb{R}^{d}\right)$. It is easy to see that $E\left(\rho_{n}\right) \rightarrow E(\rho)$ as $n \rightarrow \infty$, and hence there exists $N \in \mathbb{N}$ large enough such that $E\left(\rho_{N}\right)<\frac{1}{2} W_{\infty}$ (see the proof of Lemma 2.10 for a similar calculation). This proves the lemma with $S=N$.

Below we always consider $S>0$ to be a radius obtained from Lemma 2.5; that is, a number such that $W$ is unstable on $\mathcal{P}_{S}\left(\mathbb{R}^{d}\right)$.

The following two lemmas are fundamental in the proof of our main result. The first one shows that if, for some $R$, a point is in the support of a minimiser $\rho_{R}$ on $\mathcal{P}_{R}\left(\mathbb{R}^{d}\right)$, then there has to be at least some mass not far from it. The quantification of "some" and "not far" are independent of $R$ and the point one chooses, so that this is a uniform estimate for all minimisers and all points: 
Lemma 2.6. Suppose that the potential $W$ satisfies Hypotheses $1-5$. Then there are constants $r, m>0$ (depending only on $W$ ) such that for all $R \geqq S$ and all global minimisers $\rho_{R}$ of the energy $(1.1)$ on $\mathcal{P}_{R}\left(\mathbb{R}^{d}\right)$ we have

$$
\int_{B\left(x_{0}, r\right)} \mathrm{d} \rho_{R}(x) \geqq m \text { for all } x_{0} \in \operatorname{supp} \rho_{R} .
$$

Proof. We proceed in two steps: we first prove the result $\rho_{R}$-almost everywhere, and then everywhere in supp $\rho_{R}$.

Step 1: $\rho_{R}$-almost everywhere. Call $E_{R}$ the minimum of the energy on $\mathcal{P}_{R}\left(\mathbb{R}^{d}\right)$; that is, $E_{R}:=\min \left\{E(\rho) \mid \rho \in \mathcal{P}_{R}\left(\mathbb{R}^{d}\right)\right\}$. (We know this minimum exists due to Lemma 2.2.) Clearly $E_{R}$ is non-increasing in $R$ and $E_{R} \leqq E_{S}<\frac{1}{2} W_{\infty}$ for all $R \geqq S$ by our choice of $S$ (see Lemma 2.5). If we consider a global minimiser $\rho_{R}$ on $\mathcal{P}_{R}\left(\mathbb{R}^{d}\right)$, we know by Lemma 2.3 that for $\rho_{R}$-almost all $z \in \operatorname{supp} \rho_{R}$ we have

$$
\frac{1}{2} \int_{\mathbb{R}^{d}} W(z-x) \mathrm{d} \rho_{R}(x)=E\left(\rho_{R}\right)=E_{R} \leqq E_{S}<\frac{1}{2} W_{\infty} .
$$

Note that $E_{S}$ is independent of $R$ and of the choice of the global minimiser $\rho_{R}$. Choose $A \in \mathbb{R}$ with $E_{S}<A<\frac{1}{2} W_{\infty}$. Since by definition we have $\lim _{|x| \rightarrow \infty} W(x)$ $=W_{\infty}$, we can choose $r^{\prime}>0$ with $W(x) \geqq 2 A$ for all $x \in \mathbb{R}^{d}$ such that $|x| \geqq r^{\prime}$. (Notice that both $A$ and $r^{\prime}$ are independent of $R$.) Then for $\rho_{R}$-almost every $z$ we have

$$
\begin{aligned}
2 E_{R}= & \int_{\mathbb{R}^{d}} W(z-x) \mathrm{d} \rho_{R}(x)=\int_{B\left(z, r^{\prime}\right)} W(z-x) \mathrm{d} \rho_{R}(x) \\
& +\int_{\mathbb{R}^{d} \backslash B\left(z, r^{\prime}\right)} W(z-x) \mathrm{d} \rho_{R}(x) \\
\geqq & W_{\min } \int_{B\left(z, r^{\prime}\right)} \mathrm{d} \rho_{R}(x)+2 A \int_{\mathbb{R}^{d} \backslash B\left(z, r^{\prime}\right)} \mathrm{d} \rho_{R}(x) \\
= & \left(W_{\min }-2 A\right) \int_{B\left(z, r^{\prime}\right)} \mathrm{d} \rho_{R}(x)+2 A,
\end{aligned}
$$

where we have used that $\rho_{R}$ is a probability measure. Rearranging terms and noticing that $W_{\min }-2 A<W_{\min }-2 E_{S}<0$ and $2 E_{S} \geqq 2 E_{R}$,

$$
\int_{B\left(z, r^{\prime}\right)} \mathrm{d} \rho_{R}(x) \geqq \frac{A-E_{S}}{A-\frac{1}{2} W_{\min }}=: m .
$$

This finishes this step, since the right-hand side depends only on $W$.

Step 2: everywhere. Take $\delta>0$, call $r:=r^{\prime}+\delta$ and let $x_{0} \in \operatorname{supp} \rho_{R}$. Then we get $\rho_{R}\left(B\left(x_{0}, \delta\right)\right)>0$ by definition of the support of $\rho_{R}$. Suppose first that $\rho_{R}\left(B\left(x_{0}, \delta\right) \backslash\left\{x_{0}\right\}\right)=0$. Then $\rho_{R}\left(\left\{x_{0}\right\}\right)>0$ and therefore (2.1) has to be satisfied at $x_{0}$, so

$$
\int_{B\left(x_{0}, r\right)} \mathrm{d} \rho_{R}(x) \geqq \int_{B\left(x_{0}, r^{\prime}\right)} \mathrm{d} \rho_{R}(x) \geqq m .
$$


Suppose now that $\rho_{R}\left(B\left(x_{0}, \delta\right) \backslash\left\{x_{0}\right\}\right)>0$. Then there is $y_{0} \in B\left(x_{0}, \delta\right) \backslash\left\{x_{0}\right\}$ such that (2.1) has to be satisfied at $y_{0}$. Thus, since $B\left(y_{0}, r^{\prime}\right) \subset B\left(x_{0}, r\right)$,

$$
\int_{B\left(x_{0}, r\right)} \mathrm{d} \rho_{R}(x) \geqq \int_{B\left(y_{0}, r^{\prime}\right)} \mathrm{d} \rho_{R}(x) \geqq m .
$$

Hence the result holds for all $x_{0} \in \operatorname{supp} \rho_{R}$ as $r$ depends only on $W$.

The following lemma is in some sense complementary to the previous one: we show that if a minimiser $\rho_{R}$ on $\mathcal{P}_{R}\left(\mathbb{R}^{d}\right)$ has a "gap" in one of the coordinates, then it cannot be very big, with the quantification of "very big" being again independent of $R$ and the position of the gap. It is interesting to note that this is the only part in the paper where Hypothesis 6 is explicitly used: all later dependence on this hypothesis is through this lemma.

In order to state this precisely let us denote by $\pi_{k}: \mathbb{R}^{d} \rightarrow \mathbb{R}$ the $k$-th coordinate projection, for $k \in\{1, \ldots, d\}$. Then the following holds:

Lemma 2.7. Assume that the potential $W$ satisfies Hypotheses 1,3 and 6. Let $R \geqq 0$ and suppose that $\rho_{R}$ is a global minimiser of the energy (1.1) on $\mathcal{P}_{R}\left(\mathbb{R}^{d}\right)$. Then the support of $\rho_{R}$ cannot have "gaps" larger than $2 R_{6}$ in each coordinate (where $R_{6}$ is the constant from Hypothesis 6$)$ : if $k \in\{1, \ldots, d\}$ and $a_{k} \in \mathbb{R}$ is such that $\pi_{k}^{-1}\left(\left[a_{k}-R_{6}, a_{k}+R_{6}\right]\right) \subseteq \mathbb{R}^{d} \backslash \operatorname{supp} \rho_{R}$, then either $\pi_{k}^{-1}\left(\left(-\infty, a_{k}-R_{6}\right]\right) \subseteq$ $\mathbb{R}^{d} \backslash \operatorname{supp} \rho_{R}$ or $\pi_{k}^{-1}\left(\left[a_{k}+R_{6}, \infty\right)\right) \subseteq \mathbb{R}^{d} \backslash \operatorname{supp} \rho_{R}$.

Proof. If this is not the case, take $k \in\{1, \ldots, d\}$ and $a_{k} \in \mathbb{R}$ with $\pi_{k}^{-1}\left(\left[a_{k}-R_{6}, a_{k}\right.\right.$ $\left.\left.+R_{6}\right]\right) \subseteq \mathbb{R}^{d} \backslash \operatorname{supp} \rho_{R}$, and such that the support of $\rho_{R}$ intersects both the "left" part

$$
H_{\mathrm{L}}:=\pi_{k}^{-1}\left(\left(-\infty, a_{k}-R_{6}\right]\right)
$$

and the "right" part

$$
H_{\mathrm{R}}:=\pi_{k}^{-1}\left(\left[a_{k}+R_{6}, \infty\right)\right) .
$$

Take $0<\varepsilon_{k} \leqq R_{6}$ and $\varepsilon=\left(0, \ldots, 0, \varepsilon_{k}, 0, \ldots, 0\right) \in \mathbb{R}^{d}$ with $k$-th coordinate $\varepsilon_{k}$, and consider

$$
\tilde{\rho}_{R}:=\left.\rho_{R}\right|_{H_{\mathrm{L}}}+T_{\varepsilon}\left(\left.\rho_{R}\right|_{H_{\mathrm{R}}}\right),
$$

where $\left.\mu\right|_{A}$ denotes the restriction of a measure $\mu$ to a set $A, T_{\varepsilon}(\mu)$ the push-forward of a measure $\mu$ by the $\varepsilon$-translation $T_{\varepsilon}: x \mapsto x-\varepsilon$, and as usual $\chi_{A}$ denotes the characteristic function of a set $A$. Clearly $\tilde{\rho}_{R} \in \mathcal{P}_{R}\left(\mathbb{R}^{d}\right)$ and it is the result of slightly moving to the "left" the part of $\rho_{R}$ in the $k$-coordinate which is to the "right" of $a_{k}+R_{6}$. By Hypotheses 3 and $6, \tilde{\rho}_{R}$ has lower energy than $\rho_{R}$ :

$$
\begin{aligned}
E\left(\tilde{\rho}_{R}\right)= & E\left(\left.\rho_{R}\right|_{H_{\mathrm{L}}}\right)+E\left(T_{\varepsilon}\left(\left.\rho_{R}\right|_{H_{\mathrm{R}}}\right)\right) \\
& +\left.\int_{\mathbb{R}^{d}} \int_{\mathbb{R}^{d}} W(x-y) \mathrm{d} \rho_{R}\right|_{H_{\mathrm{L}}}(x) \mathrm{d} T_{\varepsilon}\left(\left.\rho_{R}\right|_{H_{\mathrm{R}}}\right)(y)
\end{aligned}
$$




$$
\begin{aligned}
& =E\left(\left.\rho_{R}\right|_{H_{\mathrm{L}}}\right)+E\left(\left.\rho_{R}\right|_{H_{\mathrm{R}}}\right)+\left.\left.\int_{\mathbb{R}^{d}} \int_{\mathbb{R}^{d}} W(x-y-\varepsilon) \mathrm{d} \rho_{R}\right|_{H_{\mathrm{L}}}(x) \mathrm{d} \rho_{R}\right|_{H_{\mathrm{R}}}(y) \\
& <E\left(\left.\rho_{R}\right|_{H_{\mathrm{L}}}\right)+E\left(\left.\rho_{R}\right|_{H_{\mathrm{R}}}\right)+\left.\left.\int_{\mathbb{R}^{d}} \int_{\mathbb{R}^{d}} W(x-y) \mathrm{d} \rho_{R}\right|_{H_{\mathrm{L}}}(x) \mathrm{d} \rho_{R}\right|_{H_{\mathrm{R}}}(y) \\
& =E\left(\rho_{R}\right) .
\end{aligned}
$$

Notice that we use here the translation invariance of the energy $E$ and the fact that

$$
\rho_{R}=\left.\rho_{R}\right|_{H_{\mathrm{L}}}+\left.\rho_{R}\right|_{H_{\mathrm{R}}} .
$$

The strict inequality is due to $W$ being strictly increasing in the $k$-th coordinate on $\mathbb{R}^{k-1} \times\left[2 R_{6}-\varepsilon_{k}, \infty\right) \times \mathbb{R}^{d-k}$ and to our assumption that the support of $\rho_{R}$ intersects both $H_{\mathrm{L}}$ and $H_{\mathrm{R}}$. This contradicts the fact that $\rho_{R}$ is a global minimiser on $\mathcal{P}_{R}\left(\mathbb{R}^{d}\right)$.

Remark 2.8. Notice that the proof of Lemma 2.7 still works if instead of Hypothesis 6 we suppose the following, less restrictive, growth assumption: for every $k \in$ $\{1, \ldots, d\}$ there exists $0<\varepsilon_{k} \leqq R_{6}$ such that if $x \in \mathbb{R}^{d}$ is with $x_{k} \geqq R_{6}$, then $W(x+\varepsilon)>W(x)$, where $\varepsilon=\left(0, \ldots, 0, \varepsilon_{k}, 0, \ldots, 0\right) \in \mathbb{R}^{d}$ with $k$-th coordinate $\varepsilon_{k}$. This implies that all our results using Hypothesis 6 stay true by relaxing it to this growth assumption (in particular Theorem 1.4).

Next we are able to give a uniform bound on the diameter of a minimiser $\rho_{R}$ on $\mathcal{P}_{R}\left(\mathbb{R}^{d}\right)$ which is independent of $R$. This already contains the main part of the proof of existence of a global minimiser, since it could be used, for example, to show the tightness of a minimising sequence. It is the main ingredient in Lemma 2.10, which is the existence part of Theorem 1.4.

Lemma 2.9. Assume that the potential $W$ satisfies Hypotheses 1-6. There exists $K>0$ (depending only on $W$ and $d$ ) such that for all $R \geqq 0$ and global minimiser $\rho_{R}$ of the energy (1.1) on $\mathcal{P}_{R}\left(\mathbb{R}^{d}\right)$, the diameter of the support of $\rho_{R}$ is bounded by $K$.

Proof. Let $S$ be a radius given by Lemma 2.5 (that is such that $W$ is unstable on $\mathcal{P}_{R}\left(\mathbb{R}^{d}\right)$ for all $\left.R \geqq S\right)$. Since $S$ depends only on $W$, it is clearly enough to show the lemma for $R \geqq S$.

Take any $x_{0} \in \operatorname{supp} \rho_{R}$. We recursively define $N+1$ points $\left\{x_{0}, \ldots, x_{N}\right\}$, for some $N \geqq 0$, as follows:

1. If supp $\rho_{R} \backslash \bigcup_{i=0}^{n-1} B\left(x_{i}, 2 r\right)$, where $r$ is the constant in Lemma 2.6 and $n$ is the number of already selected points, is not empty, then take any $x_{n}$ in that set.

2. If the above set is empty, then $x_{n-1}$ is the last term of the sequence (that is $N=n-1)$.

We notice that this process must end after at most $\lceil 1 / m\rceil$ steps; this is, $N+1 \leqq$ $\lceil 1 / m\rceil$, where $m$ is the constant in Lemma 2.6 and $\lceil\cdot\rceil$ is the ceiling function. The reason for this is that, for each $i \in\{0 \ldots, N\}$, the ball $B\left(x_{i}, r\right)$ contains at least a fixed amount $m$ of mass (see Lemma 2.6), and this mass is not in any of the other balls. Also, it is clear that the support of $\rho_{R}$ is contained in $\bigcup_{i=0}^{N} B\left(x_{i}, 2 r\right)$. 
We write $x_{i}=\left(x_{i}^{(1)}, \ldots, x_{i}^{(d)}\right)$ for all $i \in\{0 \ldots, N\}$, and for any $k \in\{1, \ldots, d\}$ we relabel the points so that $x_{0}^{(k)}<\cdots<x_{N}^{(k)}$. Then, if $N>0$, we have

$$
x_{i+1}^{(k)}-x_{i}^{(k)} \leqq 4 r+2 R_{6}
$$

for all $i \in\{0 \ldots, N-1\}$, due to Lemma 2.7 (otherwise the support of $\rho_{R}$ would have a gap larger than $2 R_{6}$ in the $k$-th coordinate). From this we deduce that

$$
x_{N}^{(k)}-x_{0}^{(k)} \leqq N\left(4 r+2 R_{6}\right) \leqq(\lceil 1 / m\rceil-1)\left(4 r+2 R_{6}\right) .
$$

Note that this inequality still holds if $N=0$, as in this case $m$ must be 1 . Since $k$ is arbitrary, we have that the diameter of the support of $\rho_{R}$ in each coordinate $k$ is bounded by $4 r+(\lceil 1 / m\rceil-1)\left(4 r+2 R_{6}\right)$. Therefore the diameter of the support of $\rho_{R}$ with respect to the 2 -Euclidean norm satisfies

$$
\operatorname{diam}\left(\operatorname{supp} \rho_{R}\right) \leqq \sqrt{d}\left(4 r+(\lceil 1 / m\rceil-1)\left(4 r+2 R_{6}\right)\right)=: K .
$$

Note that $K$ does not depend on $R$ or on the choice of $\rho_{R}$.

\subsection{Minimisers on the Whole Set of Probability Measures}

We now finish the proof of the existence part of Theorem 1.4:

Lemma 2.10. Assume that the potential $W$ satisfies Hypotheses 1-6. Then there exists a global minimiser for the energy (1.1) on $\mathcal{P}\left(\mathbb{R}^{d}\right)$.

Proof. Let $K$ be the bound on the diameter of minimisers on $\mathcal{P}_{R}\left(\mathbb{R}^{d}\right)$ for all $R \geqq 0$ given by Lemma 2.9 , and consider $\rho^{\prime}$ a global minimiser on $\mathcal{P}_{K}\left(\mathbb{R}^{d}\right)$. We show below that $\rho^{\prime}$ is in fact a global minimiser in all of $\mathcal{P}\left(\mathbb{R}^{d}\right)$.

Given $\rho \in \mathcal{P}\left(\mathbb{R}^{d}\right)$ with compact support, there exists $R \geqq 0$ such that $\rho \in$ $\mathcal{P}_{R}\left(\mathbb{R}^{d}\right)$. Let us take $\rho_{R}$ a global minimiser of $E$ on $\mathcal{P}_{R}\left(\mathbb{R}^{\bar{d}}\right)$. Then, we have $E\left(\rho_{R}\right) \leqq E(\rho)$. Due to translation invariance of $E$, it is clear that $E\left(\rho^{\prime}\right) \leqq E\left(\rho_{R}\right)$ for any $R \geqq 0$ since the support of $\rho$ must have diameter less than $K$, and then it can be translated to a measure in $\mathcal{P}_{K}\left(\mathbb{R}^{d}\right)$. Therefore, we conclude that $E\left(\rho^{\prime}\right) \leqq E(\rho)$ for all $\rho \in \mathcal{P}\left(\mathbb{R}^{d}\right)$ with compact support.

We want now to show that $\rho^{\prime}$ is in fact a global minimiser on $\mathcal{P}\left(\mathbb{R}^{d}\right)$. Take any $\rho \in \mathcal{P}\left(\mathbb{R}^{d}\right)$. For $n$ large enough such that $M_{n}:=\rho(B(0, n))>0$, let us define the sequence $\left(\rho_{n}\right)_{n}$ by

$$
\rho_{n}=\frac{1}{M_{n}} \chi_{B(0, n)} \rho
$$

Then

$$
\begin{aligned}
E\left(\rho_{n}\right)-\frac{W_{\min }}{2} & =\frac{1}{2} \int_{\mathbb{R}^{d} \times \mathbb{R}^{d}}\left(W(x-y)-W_{\min }\right) \mathrm{d} \rho_{n}(x) \mathrm{d} \rho_{n}(y) \\
& =\frac{1}{2 M_{n}^{2}} \int_{\mathbb{R}^{d} \times \mathbb{R}^{d}} \chi_{B(0, n)^{2}}(x, y)\left(W(x-y)-W_{\min }\right) \mathrm{d} \rho(x) \mathrm{d} \rho(y) .
\end{aligned}
$$


Applying the Lebesgue monotone convergence theorem, we get

$$
E\left(\rho_{n}\right) \underset{n \rightarrow \infty}{\longrightarrow} \frac{1}{2} \int_{\mathbb{R}^{d} \times \mathbb{R}^{d}} W(x-y) \mathrm{d} \rho(x) \mathrm{d} \rho(y)=E(\rho) .
$$

Moreover, since $\rho_{n} \in \mathcal{P}_{n}\left(\mathbb{R}^{d}\right)$ for all $n$ large enough has compact support, we have by above that $E\left(\rho_{n}\right) \geqq E\left(\rho^{\prime}\right)$. Hence $E(\rho) \geqq E\left(\rho^{\prime}\right)$. Therefore $\rho^{\prime}$ is a global minimiser on $\mathcal{P}\left(\mathbb{R}^{d}\right)$.

\subsection{Support Compactness of Minimisers}

The previous section shows the existence of a compactly supported global minimiser among all probability measures. However, this does not exclude the existence of a global minimiser without compact support. Corollary 1.5 shows that any global minimiser on $\mathcal{P}\left(\mathbb{R}^{d}\right)$ is actually compactly supported (and hence, due to Lemma 2.9, has support with diameter less than or equal to $K$ ), thus finishing the proof of Theorem 1.4. Its proof is based on very similar reasonings used throughout Lemma 2.6.

Proof of Corollary 1.5. By Remark 2.4 and Hypothesis 4 we have that $W * \rho=$ $2 E(\rho) \rho$-almost everywhere and we can take $A^{\prime}$ such that $E(\rho)<A^{\prime}<\frac{1}{2} W_{\infty}$. Then, similarly to the proof of Lemma 2.6, we show that for all $x_{0} \in \operatorname{supp} \rho$,

$$
\int_{B\left(x_{0}, r^{\prime \prime}\right)} \mathrm{d} \rho(x) \geqq \frac{A^{\prime}-E(\rho)}{A^{\prime}-\frac{1}{2} W_{\min }}=: m^{\prime}>0,
$$

where $r^{\prime \prime}$ can be found as in the proof of Lemma 2.6. The result follows immediately from a contradiction argument. Indeed, suppose that $\rho$ is not compactly supported. Then we can choose a sequence of $\left\lceil 1 / m^{\prime}\right\rceil+1$ points in its support, where $\lceil\cdot\rceil$ is the ceiling function, such that the balls with centres these points and radii $r^{\prime \prime}$ do not intersect. By the inequality above this implies that the total mass of $\rho$ is greater than 1 , contradicting the fact that $\rho$ is a probability measure.

\subsection{Corollary for Local Minimisers}

Under Hypotheses 1-6, Theorem 1.4 trivially ensures the existence of compactly supported local minimisers in any topology. However, it is not sufficient to show that any local minimiser must have compact support. Let us restrict ourselves to local minimisers with respect to the quadratic Wasserstein distance $d_{2}$ (for a definition, see [3, Section 2] for example). We know by [3, Theorem 4(i)] that, under Hypotheses 1-3 and 5, if $\rho \in \mathcal{P}\left(\mathbb{R}^{d}\right)$ is a $d_{2}$-local minimiser with $E(\rho)<+\infty$, then it satisfies $W * \rho=2 E(\rho) \rho$-almost everywhere: that is, Lemma 2.3 (and Remark 2.4) is true for $d_{2}$-local minimisers on $\mathcal{P}\left(\mathbb{R}^{d}\right)$. We give here a generalisation of Corollary 1.5 by restricting the instability condition of Theorem 1.4 to the subset of $d_{2}$-local minimisers: 
Corollary 2.11. Assume that $W: \mathbb{R}^{d} \rightarrow \mathbb{R} \cup\{+\infty\}$ satisfies Hypotheses $1-3$ and 5 , and is such that $W_{\infty}:=\lim _{|x| \rightarrow \infty} W(x)$ exists (being possibly equal to $+\infty$ ). Suppose moreover that there exists a $d_{2}$-local minimiser $\rho \in \mathcal{P}\left(\mathbb{R}^{d}\right)$ of the energy (1.1) with $E(\rho)<\frac{1}{2} W_{\infty}$. Then $\rho$ is compactly supported.

Proof. The proof is direct by following the arguments of the proof of Corollary 1.5 given in Section 2.4, and using the result [3, Theorem 4(i)] for $d_{2}$-local minimisers and the fact that $E(\rho)<\frac{1}{2} W_{\infty}$.

Remark 2.12. For potentials with $\lim _{|x| \rightarrow \infty} W(x)=+\infty$, the instability condition of Corollary 2.11 is automatically verified by any non-trivial $d_{2}$-local minimiser (that is a local minimiser with finite energy). This is the case, for instance, of the power-law potential given in Proposition 3.2(i) when $a \geqq 0$.

\section{Examples and Non-Existence of Minimisers}

\subsection{Examples of Potentials With Minimisers}

We want to give explicit examples of potentials $W$ satisfying the hypotheses of Theorem 1.4. To this end we first state a lemma which gives sufficient conditions for a potential to be unstable, and therefore for Hypothesis 4 to hold. A similar result can be found in [43, Section 3.2], where alternative conditions are also given for a potential to be unstable. In the following the subscripts + and - stand for positive and negative part, respectively.

Lemma 3.1. Let $W$ be a potential satisfying Hypotheses 1 and 2, and assume furthermore that $W_{\infty}:=\lim _{|x| \rightarrow \infty} W(x)$ exists (being possibly equal to $+\infty$ ).

(i) If $W_{\infty}=+\infty$, then $W$ is unstable.

(ii) If $W_{\infty}<+\infty$, call $\tilde{W}:=W-W_{\infty}$. If $\tilde{W}_{+}$is integrable and $\int_{\mathbb{R}^{d}} \tilde{W}<0$ (being possibly equal to $-\infty)$, then $W$ is unstable.

Proof. (i) This case is trivial since, by Hypothesis 2, any uniform distribution on a given ball has finite energy.

(ii) Let us define, for all $R>0$, the following probability measure:

$$
\rho_{R}=\frac{1}{|B(0, R)|} \chi_{B(0, R)} .
$$

Then compute easily

$$
E\left(\rho_{R}\right)-\frac{1}{2} W_{\infty}=\frac{1}{2|B(0, R)|} \int_{\mathbb{R}^{d}} \phi_{R}(x) \tilde{W}(x) \mathrm{d} x,
$$

where $\phi_{R}:=\frac{1}{|B(0, R)|} \chi_{B(0, R)} * \chi_{B(0, R)}$. Remark that $\phi_{R} \leqq 1$ for all $R>0$ and $\left(\phi_{R} \tilde{W}\right)_{R>0}$ converges pointwise to $\tilde{W}$ on $\mathbb{R}^{d}$ as $R \rightarrow \infty$. Assume first that $\tilde{W}_{-}$is 
integrable, that is by our hypothesis on $\tilde{W}_{+}, \tilde{W}$ is integrable. Then, by the Lebesgue dominated convergence theorem we get

$$
\int_{\mathbb{R}^{d}} \phi_{R}(x) \tilde{W}(x) \mathrm{d} x \underset{R \rightarrow \infty}{\longrightarrow} \int_{\mathbb{R}^{d}} \tilde{W}(x) \mathrm{d} x<0 .
$$

Thus there exists $R$ large enough such that $E\left(\rho_{R}\right)<\frac{1}{2} W_{\infty}$, which shows the results. Now assume that $\tilde{W}_{-}$is not integrable, that is $\int_{\mathbb{R}^{d}} \tilde{W}=-\infty$. By above we have

$$
\begin{aligned}
E\left(\rho_{R}\right)-\frac{1}{2} W_{\infty} & =\frac{1}{2|B(0, R)|}\left(\int_{\mathbb{R}^{d}} \phi_{R}(x) \tilde{W}_{+}(x) \mathrm{d} x+\int_{\mathbb{R}^{d}} \phi_{R}(x) \tilde{W}_{-}(x) \mathrm{d} x\right) \\
& \leqq \frac{1}{2|B(0, R)|}\left(\int_{\mathbb{R}^{d}} \tilde{W}_{+}(x) \mathrm{d} x+\int_{\mathbb{R}^{d}} \phi_{R}(x) \tilde{W}_{-}(x) \mathrm{d} x\right) .
\end{aligned}
$$

Since $\left(\phi_{R} \tilde{W}_{-}\right)_{R>0}$ is non-increasing, non-positive and converges pointwise to $\tilde{W}_{-}$ on $\mathbb{R}^{d}$ as $R \rightarrow \infty$, the Lebesgue monotone convergence theorem yields

$$
\int_{\mathbb{R}^{d}} \phi_{R}(x) \tilde{W}_{-}(x) \mathrm{d} x \underset{R \rightarrow \infty}{\longrightarrow} \int_{\mathbb{R}^{d}} \tilde{W}_{-}(x) \mathrm{d} x=-\infty .
$$

Therefore, since $\int_{\mathbb{R}^{d}} \tilde{W}_{+}$is finite, there exists $R$ large enough such that $E\left(\rho_{R}\right)<$ $\frac{1}{2} W_{\infty}$, which ends the proof.

In the following proposition we use the result above to find explicit potentials satisfying all Hypotheses 1-6, and therefore for which Theorem 1.4 is applicable.

Proposition 3.2. Consider the following potentials for all $x \in \mathbb{R}^{d}$ and $C_{A}, C_{R}, \ell_{A}$, $\ell_{R}>0$ :

(i) (Power-law potential) $W(x)=\frac{|x|^{a}}{a}-\frac{|x|^{b}}{b}$ with $-d<b<a$,

(ii) (Morse potential) $W(x)=C_{R} e^{-\frac{|x|}{\ell_{R}}}-C_{A} e^{-\frac{|x|}{\ell_{A}}}$ with $\ell_{R}<\ell_{A}$ and $\frac{C_{R}}{C_{A}}<\left(\frac{\ell_{A}}{\ell_{R}}\right)^{d}$, with the convention $\frac{|x|^{0}}{0}=\log |x|$. Each of these potentials satisfies Hypotheses 1 to 6 .

Proof. (i) Hypotheses 3 and 5 are trivially respected, as well as Hypotheses 2 and 6 since $-d<b<a$. Furthermore, since $a>b$, we have that $W$ satisfies Hypothesis 1 . We are only left to show Hypothesis 4. Let us first assume $a \geqq 0$. Then $W(x) \rightarrow+\infty=: W_{\infty}$ as $|x| \rightarrow \infty$. Therefore, by Lemma 3.1(i) we have that $W$ satisfies Hypothesis 4. On the other hand, in the case $a<0$ we have $W(x) \rightarrow 0=: W_{\infty}$ as $|x| \rightarrow \infty$. Since $a>b, W$ is asymptotic to $-|x|^{b} / b$ as $|x| \rightarrow \infty$, while $W_{+}$is integrable since $-d<a$. This shows that $\int_{\mathbb{R}^{d}} W=-\infty$, so Lemma 3.1(ii) applies to show that $W$ satisfies Hypothesis 4.

(ii) Hypotheses 2, 3 and 5 are trivially respected, as well as Hypotheses 1 and 6 by our assumptions on the parameters. Furthermore $W(x) \rightarrow 0=: W_{\infty}$ as $|x| \rightarrow \infty$, and one may check that

$$
\int_{\mathbb{R}^{d}} W(x) \mathrm{d} x=C^{\prime} \Gamma(d)\left(C_{R} \ell_{R}^{d}-C_{A} \ell_{A}^{d}\right),
$$


where $C^{\prime}>0$ is a constant coming from a spherical change of variables and $\Gamma$ is the Gamma-function. Therefore $\int_{\mathbb{R}^{d}} W<0$ for the given range of parameters, and by Lemma 3.1(ii) we obtain that $W$ satisfies Hypothesis 4 .

\subsection{Non-Existence of Minimisers}

In the work of SimIONE et al. [45] conditions are given for the non-existence of minimisers of the interaction energy. Here, for completeness, we rewrite their result in Theorem 3.3 adapting it to our hypotheses and using a slightly different language. Let us consider the following assumption on the potential:

Hypothesis 7. The limit (1.5) exists and $W$ is such that there is $\rho \in \mathcal{P}\left(\mathbb{R}^{d}\right)$ with $E(\rho) \leqq \frac{1}{2} W_{\infty}$.

This is a formulation of Hypothesis 4 generalised to the equality case. Our main result, as given in Theorem 1.4, can now be extended to the following:

Theorem 3.3. Suppose that the potential $W$ satisfies Hypotheses 1-3, 5 and 6. Also assume it is such that the limit (1.5) exists and the positive part of $\tilde{W}:=W-W_{\infty}$ is integrable if $W_{\infty}<+\infty$. Then $E$ admits a global minimiser if and only if $W$ satisfies Hypothesis 7.

Proof. Sufficiency: The sufficiency is almost direct by Theorem 1.4. However we still need to cover the equality case. By contradiction, suppose that there is $\rho \in$ $\mathcal{P}\left(\mathbb{R}^{d}\right)$ with $E(\rho)=\frac{1}{2} W_{\infty}$ and that there exists no global minimiser of the energy. Then there must be a probability measure $\rho^{\prime}$ such that $E\left(\rho^{\prime}\right)<E(\rho)=\frac{1}{2} W_{\infty}$. Now we can apply Theorem 1.4 to get that there exists a global minimiser for $E$, which contradicts the non-existence assumption.

Necessity: Suppose that there exists a global minimiser $\rho^{\prime} \in \mathcal{P}\left(\mathbb{R}^{d}\right)$ for the energy (1.1). If $W_{\infty}=+\infty$, then the result is trivial. Assume that $W_{\infty}<+\infty$. By definition of $W_{\infty}$, we have $\lim _{|x| \rightarrow \infty} \tilde{W}(x)=0$. Then, since $W$ satisfies Hypothesis 6 , we know there exists $r>0$ large enough such that $\tilde{W}(x) \leqq 0$ for all $x \in \mathbb{R}^{d}$ such that $|x|>r$. Thus we know that $\int_{\mathbb{R}^{d}} \tilde{W} \neq+\infty$ by Hypothesis 2 . Now, since $\tilde{W}_{+}$is integrable, by proceeding as in the proof of Lemma 3.1 and using the same notation, we have two cases: either $\int_{\mathbb{R}^{d}} \tilde{W}$ is finite and therefore $E\left(\rho_{R}\right) \rightarrow \frac{1}{2} W_{\infty}$ as $R \rightarrow \infty$, or $\int_{\mathbb{R}^{d}} \tilde{W}=-\infty$ and therefore there is $R$ large enough such that $E\left(\rho_{R}\right)<\frac{1}{2} W_{\infty}$. In both cases we get $E\left(\rho^{\prime}\right) \leqq \frac{1}{2} W_{\infty}$. Hence result.

Remark 3.4. Theorem 3.3 shows that, under its hypotheses, the only stable potentials for which global minimisers exist are the ones such that the equality case in the stability definition holds, that is the ones such that you can find $\rho \in \mathcal{P}\left(\mathbb{R}^{d}\right)$ with $E(\rho)=\frac{1}{2} W_{\infty}$. For radially symmetric potentials this is also true without Hypothesis 6, as proven in [45]. An example of such potential is the following:

$$
W(x)=|x|^{2} e^{-|x|^{2}}, \quad x \in \mathbb{R}^{d} .
$$

Indeed $W$ is radially symmetric and satisfies all the hypotheses of Theorem 3.3 but Hypothesis 6 , and is stable with obviously $E\left(\delta_{0}\right)=\frac{1}{2} W(0)=0=\frac{1}{2} W_{\infty}$, where $\delta_{0}$ is the Dirac measure centred at the origin. 
Acknowledgments The authors would like to thank D. Chafaï for mentioning some references regarding the circular law in probability. J. A. Cañizo acknowledges support from projects MTM2011-27739-C04-02 and the Marie-Curie CIG project KineticCF. J. A. Carrillo acknowledges support from projects MTM2011-27739-C04-02, the Royal Society through a Wolfson Research Merit Award, and the Engineering and Physical Sciences Research Council (UK) Grant number EP/K008404/1.

Open Access This article is distributed under the terms of the Creative Commons Attribution 4.0 International License (http://creativecommons.org/licenses/by/4.0/), which permits unrestricted use, distribution, and reproduction in any medium, provided you give appropriate credit to the original author(s) and the source, provide a link to the Creative Commons license, and indicate if changes were made.

\section{References}

1. Albi, G., Balagué, D., Carrillo, J.A., von Brecht, J.: Stability analysis of flock and mill rings for 2nd order models in swarming. SIAM J. Appl. Math. (2014) (to appear)

2. Au Yeung, Y., Friesecke, G., Schmidt, B.: Minimizing atomic configurations of short range pair potentials in two dimensions: crystallization in the Wulff shape. Calc. Var. Partial Differ. Equ., 44(1-2), 81-100 (2012)

3. Balagué, D., Carrillo, J.A., Laurent, T., Raoul, G.: Dimensionality of local minimizers of the interaction energy. Arch. Rational Mech. Anal., 209(3), 1055-1088 (2013)

4. Balagué, D., Carrillo, J.A., Laurent, T., Raoul, G.: Nonlocal interactions by repulsive-attractive potentials: radial ins/stability. Phys. D 260, 5-25 (2013)

5. Balagué, D., Carrillo, J.A., Yao, Y.: Confinement for repulsive-attractive kernels. Discrete Contin. Dyn. Syst. Ser. B 19(5), 1227-1248 (2014)

6. BäUerle, N., Rieder, U.: Markov Decision Processes with Applications to Finance, 1st edn. Springer, Berlin, 2011

7. Bavaud, F.: Equilibrium properties of the Vlasov functional: The generalized PoissonBoltzmann-Emden equation. Rev. Modern Phys. 63(1), 129+ (1991)

8. Benedetto, D., Caglioti, E., Carrillo, J.A., Pulvirenti, M.: A non-maxwellian steady distribution for one-dimensional granular media. J. Stat. Phys. 91, 979-990 (1998)

9. Bernoff, A.J., Topaz, C.M.: A primer of swarm equilibria. SIAM J. Appl. Dyn. Syst. 10(1), 212-250 (2011)

10. Bertozzi, A.L., Carrillo, J.A., Laurent, T.: Blow-up in multidimensional aggregation equations with mildly singular interaction kernels. Nonlinearity 22(3), 683-710 (2009)

11. Bertozzi, A.L., Laurent, T., LÉGer, F.: Aggregation and spreading via the Newtonian potential: the dynamics of patch solutions. Math. Models Methods Appl. Sci. 22(supp01), $1140005+(2012)$

12. Blanchet, A., Carlier, G.: From Nash to Cournot-Nash equilibria via the MongeKantorovich problem. TSE Working Paper, pp. 14-490 2014

13. Caffarelli, L., Vázquez, J.L.: Asymptotic behaviour of a porous medium equation with fractional diffusion. Discrete Contin. Dyn. Syst. Ser. A 29(4), 1393-1404 (2011)

14. Caffarelli, L.A., VÁzQuez, J.L.: Nonlinear porous medium flow with fractional potential pressure 2011

15. CARrillo, J.A., Chertock, A., HuAng, Y.: A finite-volume method for nonlinear nonlocal equations with a gradient flow structure 2014 (Preprint)

16. Carrillo, J.A., Delgadino, M.G., Mellet, A.: Regularity of local minimizers of the interaction energy via obstacle problems. (2014) (Preprint)

17. Carrillo, J.A., DiFrancesco, M., Figalli, A., Laurent, T., Slepčev, D.: Globalin-time weak measure solutions and finite-time aggregation for nonlocal interaction equations. Duke Math. J. 156(2), 229-271 (2011) 
18. Carrillo, J.A., Ferreira, L.C.F., Precioso, J.C.: A mass-transportation approach to a one dimensional fluid mechanics model with nonlocal velocity. Adv. Math. 231(1), 306-327 (2012)

19. Carrillo, J.A., Huang, Y., Martin, S.: Explicit flock solutions for quasi-morse potentials. Eur. J. Appl. Math. (2014) (to appear)

20. Carrillo, J.A., Huang, Y., Martin, S.: Nonlinear stability of flock solutions in secondorder swarming models. Nonlinear Anal. Real World Appl. 17, 332-343 (2014)

21. Carrillo, J.A., Martin, S., Panferov, V.: A new interaction potential for swarming models. Phys. D 260, 112-126 (2013)

22. Carrillo, J.A., McCann, R.J., Villani, C.: Kinetic equilibration rates for granular media and related equations: entropy dissipation and mass transportation estimates. Rev. Mat. Iberoamericana 19(3), 971-1018 (2003)

23. Carrillo, J.A., McCann, R.J., Villani, C.: Contractions in the 2-Wasserstein length space and thermalization of granular media. Arch. Ration. Mech. Anal. 179(2), 217-263 (2006)

24. Chafaï, D., Gozlan, N., Zitt, P.-A.: First order global asymptotics for confined particles with singular pair repulsion. arXiv:1304.7569v3 (2013)

25. Choksi, R., Fetecau, R.C., Topaloglu, I.: On minimizers of interaction functionals with competing attractive and repulsive potentials 2014 (Preprint)

26. Degond, P., Liu, J.-G., Ringhofer, C.: Evolution of the distribution of wealth in an economic environment driven by local Nash equilibria. J. Stat. Phys. 154, 751-780 (2014)

27. D’Orsogna, M.R., Chuang, Y.L., Bertozzi, A.L., Chayes, L.S.: Self-propelled particles with soft-core interactions: patterns, stability, and collapse. Phys. Rev. Lett. 96(10), $104302+(2006)$

28. Doye, J.P.K., Wales, D.J., Berry, R.S.: The effect of the range of the potential on the structures of clusters. J. Chem. Phys. 103, 4234-4249 (1995)

29. E, W., Li, D.: On the crystallization of 2D hexagonal lattices. Comm. Math. Phys. 286(3), 1099-1140 (2009)

30. Fellner, K., Raoul, G.: Stable stationary states of non-local interaction equations. Math. Models Methods Appl. Sci. 20(12), 2267-2291 (2010)

31. Fellner, K., Raoul, G.: Stability of stationary states of non-local equations with singular interaction potentials. Math. Comput. Modelling 53(7-8), 1436-1450 (2011)

32. Fetecau, R., Huang, Y., Kolokolnikov, T.: Swarm dynamics and equilibria for a nonlocal aggregation model. Nonlinearity 24(10), 2681-2716 (2011)

33. Fetecau, R.C., Huang, Y.: Equilibria of biological aggregations with nonlocal repulsiveattractive interactions. Physica D: Nonlinear Phenomena (2012)

34. Fisher, M.E., Ruelle, D.: The stability of Many-Particle systems. J. Math. Phys. 7(2), 260-270 (1966)

35. Fonseca, I., Müller, S.: A uniqueness proof for the Wulff theorem. Proc. R. Soc. Edinburgh Sect. A 119(1-2), 125-136 (1991)

36. Frostman, O.: Potentiel d'équilibre et capacité des ensembles. $\mathrm{PhD}$ thesis, University of Lund (1935)

37. Hagan, M.F., Chandler, D.: Dynamic pathways for viral capsid assembly. Biophys. J. 91, 42-54 (2006)

38. Kolokolnikov, T., Carrillo, J.A., Bertozzi, A., Fetecau, R., Lewis, M.: Emergent behaviour in multi-particle systems with non-local interactions (Editorial). Phys. D 260, $1-4(2013)$

39. Li, H., Toscani, G.: Long-time asymptotics of kinetic models of granular flows. Arch. Ration. Mech. Anal. 172(3), 407-428 (2004)

40. López García, A.: Greedy energy points with external fields. In: Arvesú, J., MarcelLÁn, F., Martínez-Finkelshtein, A. (ed.) Recent Trends in Orthogonal Polynomials and Approximation Theory, vol. 507, pp. 189-207. American Mathematical Society, Providence 2010 
41. Raoul, G.: Nonlocal interaction equations: stationary states and stability analysis. Differ. Int. Equ. 25(5-6), 417-440 (2012)

42. Rechtsman, M.C., Stillinger, F.H., Torquato, S.: Optimized interactions for targeted self-assembly: application to a honeycomb lattice. Phys. Rev. Lett. 95(22), (2005)

43. Ruelle, D.: Statistical mechanics: rigorous results, 1st edn. W.A. Benjamin, New York (1969)

44. SAFF, E.B., Totik, V.: Logarithmic potentials with external fields, vol. 316 of Grundlehren der mathematischen Wissenchaften. Springer Berlin Heidelberg, Berlin, Heidelberg (1997)

45. Simione, R., SlepČEv, D., Topaloglu, I.: Existence of minimizers of nonlocal interaction energies 2014 (Preprint)

46. Theil, F.: A proof of crystallization in two dimensions. Comm. Math. Phys. 262(1), 209-236 (2006)

47. vON BRECHT, J.H., UMINSKY, D.: On soccer balls and linearized inverse statistical mechanics. J. Nonlinear Sci. 22(6), 935-959 (2012)

48. von Brecht, J., Uminsky, D., Kolokolnikov, T., Bertozzi, A.: Predicting pattern formation in particle interactions. Math. Mod. Meth. Appl. Sci. 22, 1140002 (2012)

49. Wales, D.J.: Energy landscapes of clusters bound by short-ranged potentials. Chem. Eur. J. Chem. Phys. 11, 2491-2494 (2010)

School of Mathematics, Watson Building,

University of Birmingham,

Edgbaston, Birmingham, B152TT,

UK.

e-mail: j.a.canizo@bham.ac.uk

and

Department of Mathematics, Imperial College London,

South Kensington Campus, London, SW7 2AZ, UK.

e-mail: carrillo@imperial.ac.uk e-mail: f.patacchini13@imperial.ac.uk

(Received May 25, 2014 / Accepted February 12, 2015)

Published online March 4, 2015 - (c) The Author(s) (2015)

This article is published with open access at Springerlink.com 